\title{
Estrategias funcionales emergentes de promoción por parte de los ejecutivos del negocio bancario: Estudio preliminar a la Banca en Maracaibo
}

\author{
Borgucci, Emmanuel*- \\ Cubillán, Roberto** \\ * Economista. Candidato a Dr. en Ciencias Sociales (Convenio UCV-LUZ). Investigador \\ de la Facultad de Ciencias Económicas y Sociales de la Universidad del Zulia. \\ E-mail: eborqucci@yahoo.com \\ ** Economista de la división corporativa del Banco Occidental de Descuento.
}

\begin{abstract}
Resumen
En esta investigación de carácter preliminar, se trata de demostrar la existencia de estrategias funcionales emergentes por parte de los ejecutivos de negocios bancarios. Se diseñó una investigación de tipo descriptivo y se aplicó un cuestionario semiestructurado y validado con el procedimiento del pretest-test a 16 ejecutivos del área petrolera pertenecientes a 10 bancos ubicados en Maracaibo (Venezuela). Se concluye que sobre la base de una serie de habilidades personales y operativas, el ejecutivo de negocios no solamente tiene la habilidad para comunicarse, cruzar ventas o servir de asesor, sino que es una especie de departamento informal de gestión de recursos internos para alcanzar metas de captación y colocación e recursos del banco aunque en determinados momentos, como lo expresaron algunos ejecutivos de negocios, la institución se oponga consciente o inconscientemente.
\end{abstract}

Palabras clave: Estrategias emergentes, ejecutivos de negocios bancarios, banca comercial y universal, productos y servicios, estrategias de promoción.

\section{Emerging Fuctional Promotional Strategies on the Part of Comercial Bank Executives: A Preliminary Study of the Banking Sector in Maracaibo}

\begin{abstract}
This preliminary research deals with demonstrating the existence of emerging functional strategies on the part of bank business executives. The research was descriptive and a semi-structured questionnaire was validatd, pretested and applied to 16 bank executives
\end{abstract}


in the oil producing region working in 10 banks in Maracaibo (Venezuela). The conclusion was that due to a series of personal and operative abilities, these business executives not only were able to communicate, affect sales and serve as advisors, but also were a species of informal human resource management departments with the capacity to reach commercial placement and marketing goals even when business and banking executives were consciously or unconsciously opposed to the same.

Key words: Emerging functional strategies, bank business executives, commercial and universal banks, product/services, promotion strategies.

Recibido: 02-01-14. Aceptado: 03-09-04

\section{I ntroducción}

Durante los últimos quince años, la banca comercial y universal venezolana ha experimentado importantes transformaciones en su ámbito de negocios externos e internos. La banca comercial recibió los impactos tanto del entorno nacional como los del resto del mundo. En el ámbito nacional, por el cambio de las políticas económicas (especialmente la cambiaria, la apertura petrolera, la política fiscal y la monetaria), los efectos de la crisis bancaria de 1994 y los cambios en la legislación que regula el sector de intermediarios bancarios (sobre todo los referidos a la supervisión, fiscalización y control). En el ámbito internacional, por el reto que plantea la existencia de bancos cuyo campo de acción es mundial y con un enorme poder económico.

Debido a lo anterior, los bancos se han visto en la necesidad de realizar cambios en sus estructuras organizacionales, en su infraestructura de negocios y en los servicios que ofrecen al público. En el plano organizacional, los bancos comerciales han desarrollado estrategias de integración de vicepresidencias, departamentos y de una estandarización y coordinación de actividades de bajo nivel agregado (Stoner y Wankel, 1989). En cuanto a la infraestructura de negocios, después del masivo cierre de oficinas bancarias ocurridas después de la crisis de 1994, los bancos han comenzado la estrategia siguiente: 1) Dotación de sistemas de comunicación digitales satelital (Drew, 1995). 2) Incorporación de software que permita: el manejo masivo, diversificado, seguro de información y vender más productos/servicios (Lundy, 1990). 3) Rediseño de estrategias geográficas, en donde se retomó en cierta forma, la correlación entre la fijación de un índice de rentabilidad y la participación local (en el corredor vial, barrio o centro comercial) de la agencia (Fruhan, 1979 y Morgan, Severn y Severn (1995: 95). Con referencia a los servicios que se ofrecen al público, se observan pocos cambios en el soporte legal de las operaciones bancarias 
(contratos que soportan operaciones activas y pasivas). Sin embargo, se nota una mayor tendencia a combinar productos/servicios existentes así como la incorporación de conceptos financieros traídos desde el exterior adaptados al marco jurídico y las costumbres bancarias venezolanas para obtener "productos/servicios híbridos" (Pascual y González, 1998 y Mishkin, 1995).

Como producto de lo anterior, los bancos comerciales tienen mayores exigencias de personal calificado para las operaciones de atención al público y de banca corporativa. Con respecto al personal de atención al público, la exigencia se concentra en una mayor calificación del personal de mostrador y de banca personal (home banking). En cuanto al ejecutivo de negocios de la Banca Corporativa, el énfasis está dirigido a contar con un personal capaz de realizar actividades de carácter operativo, de venta y de asesoría financiera (debido a que este vendedor cuenta con una serie de características únicas) (Swan, 1991). En tal sentido los bancos invierten una gran cantidad de dinero en seleccionar, reclutar, entrenar e incentivar al personal de venta, ya que "un deficiente reclutamiento puede forzar a una organización a contratar a personas que no satisfacen realmente sus necesidades" (Anderson, Hair y Busch, 1995:213).

Se ha estudiado una variedad de factores que se han considerado como posibles determinantes en el comportamiento de ventas de un ejecutivo en general y que resumen el tipo de estrategias que desarrollan frente al público. Según Leigh (1987), Szymansky (1988), Weitz (1981) y Sujan, Sujan y Bettman (1988), la efectividad de la venta está relacionada con el conocimiento previo en esta materia adquirido por el ejecutivo tanto en el ámbito académico como en el ejercicio de su labor. Por otra parte, Leong, Busch y Roeder (1986) e IFE (1991), fundamentan en dos tipos de estructuras de conocimientos, la efectividad de las ventas: categorías estructurales acerca de la clasificación de los clientes (en función de sus motivaciones o comportamientos de compra) y secuencias de argumentaciones que incluye información acerca de secuencias de eventos y acciones que se presentan comúnmente en las situaciones de ventas. Por último, se debe considerar la forma en que el personal de venta es remunerado ya sea en función de cuotas de ventas o de cualquier otro método, ya que según Churchill, Ford y Walker (1985) y (1994) constituye el elemento más importante que la gerencia utiliza para motivar al personal.

En consecuencia basados en los planeamientos de Mintzberg (1978) y (1994), este artículo trata de demostrar la existencia de estrategias funcionales emergentes de ventas por parte de los ejecutivos de negocios bancarios, por medio de la puesta en marcha de una serie de habilidades personales y operativas. Este trabajo es una primera aproximación, al estudio de la forma en que el ejecutivo del negocio bancario capta 
clientes corporativos. Además, se busca destacar cómo sobre la base de un ordenamiento mercantil determinado y unas políticas comerciales establecidas por las direcciones de los bancos comerciales/universales, el ejecutivo de negocios combina una serie de productos/servicios existentes para ofrecerlos de una forma unitaria y/o combinada.

A tal efecto, se procedió a diseñar una investigación de tipo descriptivo y transeccional (Hernández et al, 1991:181) y se aplicó un cuestionario semiestructurado (con un nivel de confiabilidad del re-test del 95.6\%) a los 16 ejecutivos de negocios 1 del área petrolera pertenecientes a 10 bancos con sede en la ciudad de Maracaibo y del resto de Venezuela (Banco Occidental de Descuento, Caracas, Caribe, Citibank, Corpbanca, Venezolano de Crédito, Mercantil, Provincial, Unibanca y Venezuela).

La selección de Ejecutivos de Negocios en el área petrolera obedeció a las siguientes razones. En primer lugar, los ejecutivos de las empresas pertenecientes a este sector exigen una mayor diversidad de servicios a los bancos (operaciones de crédito, captación, operaciones con el exterior). En segundo lugar, exigen un mayor número de operaciones especializadas (uso de valuaciones, órdenes de pago, financiamiento en divisas o de operaciones de futuro en divisas, entre otras). En tercer lugar, la mayoría de los ejecutivos de negocios considerados para este estudio son al mismo tiempo ejecutivos en otras áreas o que son rotados regularmente (no hay que olvidar que en el negocio bancario la rotación en el puesto es una forma de adquirir conocimientos, habilidades y destrezas).

\section{Mercadeo estrat égico de servicios bancarios}

La venta como parte de las estrategias de la organización, desde una visión basada en el plan estratégico, es un conjunto de metas, objetivos básicos a largo plazo en una empresa y la adopción de cursos de acción de producción y distribución de recursos para lograr estos propósitos (Chandler, 1962; Andrews, et al, 1965, Andrews, 1971 y Ansoff, 19652). Sin embargo, este enfoque estático y focalizado en la dirección encontró en Mintzberg (1978) su principal crítico. Este autor estableció la existencia de dos tipos de planificación: la formal (o estrategia intentada) y la que surge de circunstancias no previstas. Según su criterio, con frecuencia las estrategias emergentes $\underline{3}$ tienen más éxito que las intentadas, debido a que tienen la capacidad de absorber las inflexibilidades del plan o la estructura formal de la organización.

Cuando Mitzberg (1994:13) hace alusión al término planeación estratégica, la define 
como: "Un procedimiento formalizado con la finalidad de producir un resultado articulado, bajo la forma de un sistema integrado de toma de decisiones". En otras palabras, planeación estratégica alude a la idea de formalización o descomposición de un proceso en diferentes etapas articuladas y asociadas al análisis racional. Bajo esta perspectiva, el autor considera que la planeación estratégica adolece de al menos tres deficiencias y tres falacias. El primer error que considera es el de compromiso. Según la gerencia el proceso de planificación estratégica es un esquema de cálculo en la que se define un curso de acción y el resto de la organización sigue esa ruta. Según el autor, el problema se agrava cuando la planificación estratégica se utiliza como herramienta para ejercer un mayor control sobre la organización en sus cuadros medios (operacionales) y bajos (funcionales). El segundo error está referido a que la planificación estratégica garantiza un cambio en el status quo dentro de la organización. Según Mintzberg, la implementación de la planificación en muchas empresas ha llevado a generar organizaciones más inflexibles, debido a que el cambio que se promueve es el genérico o de corte analítico y no el cambio creativo que se fundamenta en procesos de síntesis. El tercer error está referido al manejo político de la organización y surge cuando la planificación estratégica pone énfasis en favorecer procesos formales que generan controles sobre los niveles bajos e intermedios de la gerencia, surge el viejo conflicto entre la gerencia de línea y la de staff.

Entre las falacias que menciona Mintzberg se encuentran: 1) La predeterminación, 2) La separación y 3) La formalización. La primera hace referencia a la imposibilidad que tiene la planificación estratégica de predecir cambios en el entorno como los tecnológicos o el incremento general de los precios relativos. La segunda tiene que ver con la separación o el reduccionismo, es decir, comprimir la estrategia informal en una serie de pasos. El autor frente a la metáfora de ver el bosque más que los árboles, propone la metáfora de: “hallar el diamante en el campo. En otras palabras, los estrategas reales buscan en la tierra, metiendo sus manos, las ideas que necesitan" (Mintzberg, 1994: 17). En este sentido si bien se puede tener una visión de helicóptero, también es cierto que el bosque puede ser visto como una gran alfombra verde $y$, por lo tanto, no se puede visualizar claramente la complejidad del sistema. La tercera hace referencia con sus propias palabras: "The failure of strategic planning is the failure of formalization-of systems to do better in or even nearly as well as flesh and blood people. It is the failure of forecasting to predict discontinuities, of programming to provide creativity, of hard data o substitute for soft, of scheduling to handle the dynamics... The mechanical combination of information did not solve any fundamental problem that existed with human inuition" (1994: 19).

Los anteriores planteamientos son complementados con la correspondencia entre las 
competencias organizacionales y las oportunidades y/o riesgos surgidos en el entorno del negocio financiero (Hofer y Schendel, 1985). Tales oportunidades y riesgos en un mercado de referencia como el bancario sólo son posibles a través de la identificación de las necesidades de los clientes, el diseño de productos/servicios, la segmentación del mercado, el análisis de su atractivo, el establecimiento de la ventaja competitiva y el diseño de la estrategia correspondiente (Lambin, 1995).

Desde esta perspectiva, la función de un mercadeo financiero de carácter estratégicofuncional consiste en la realización de intercambios voluntarios entre oferentes (intermediarios bancarios) y demandantes de servicios financieros (personas jurídicas en este caso) (Lewis, 1991). Es decir, la realización de transacciones requiere la organización material del intercambio (la materialización de la entrega de los productos/servicios objetos del intercambio) y la organización de actividades de carácter comunicativo (el conjunto de flujos de información que deben preceder, acompañar y seguir al intercambio).

Dentro de las actividades de carácter comunicativo se encuentran las ventas personales. Quienes ejercen la función de las ventas cumplen, según Lambin (1995) y Mapfre (1996), varias funciones: la venta propiamente dicha, actividades de servicio y actividades de transmisión de información. Es decir, el vendedor en cualquier empresa es, al mismo tiempo, parte de su brazo comercial, de su sistema formal o informal de información de mercadeo e inspirador de estrategias emergentes.

En concordancia con lo anterior, Kotler (1996) y Kotler y Amstrong (1998) afirman que el énfasis de atraer clientes y lograr ventas ha dado paso a la creación de transacciones (el comercio de valores entre dos partes) y a mantener "relaciones" con el cliente (establecimiento de lazos a largo plazo entre los participantes de las transacciones). En otras palabras, no es suficiente hacer un esfuerzo por captar clientes, sino que se debe estudiar el comportamiento en materia de saldos así como las cuentas cerradas e inactivas. Esta perspectiva desde los estudios del comportamiento del consumidor bancario fue adelantada por Badoc (1976), quien planteaba que el futuro de los estudios sobre el comportamiento del consumidor bancario debería incluir el análisis de las cuentas muertas y de las razones que han provocado esa mortalidad.

Sin embargo consideramos que esta forma de visualizar el mercadeo, requiere algunas precisiones cuando se trata del mercadeo bancario:

- Los bancos cuentan con canales de distribución, que según Morgan, Severn y 
Severn (1995) se pueden dividir en: sucursales/agencias todo servicio, de servicios limitados o de servicios especializados.

- Es un negocio muy segmentado sobre la base de criterios sociodemográficos y psicográficos.

- Según Andrew (1995), en el mundo bancario el precio se refleja de dos maneras:

a) Existen algunos cargos que se aplican por los servicios facilitados, como costes de transferencia o tarifas anuales por servicio de tarjetas y b) El tipo de interés o el precio del dinero (sobre todo la tasa activa).

- Desde una perspectiva que vincula el mercadeo con los contratos bancarios (y con ello con los productos/servicios), para Mc. Goldrick y Greenland (1996), el precio de un producto o servicio representa el símbolo de aceptación de la existencia de un contrato de intercambio de dinero (aspecto real de muchos contratos bancarios) por productos/servicios financieros. En este punto, es interesante destacar las características que adquiere el intercambio. Desde el punto de vista de las operaciones pasivas (depósitos irregulares), el cliente que deposita dinero en una cuenta tiene un crédito (ya que existe una transmisión actual de la propiedad por parte del depositante al banco de un bien fungible llamado dinero) a su favor que será exigible de inmediato en los depósitos a la vista ("cuenta corriente bancaria" y "ahorro bancario") y de forma mediata en "depósitos a plazos bancarios" (Rodríguez, 1990), instrumentalizado por medio de una cuenta corriente "contable" en donde se anotan los cargos y abonos producto de las relaciones económicas entre el banco y el cliente. Cuando la operación es activa, el cliente debe devolver cantidad la equivalente del bien que ha recibido más una remuneración (interés, premio, descuento, etc.). La devolución es por medio de un bien fungible (dinero) que será entregado en efectivo y/o cargado en la "cuenta corriente bancaria" que el cliente tiene en la entidad bancaria.

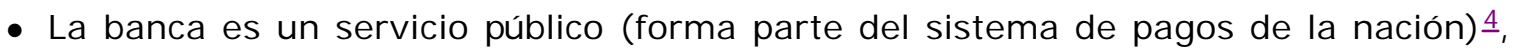
sujeto a efectos de agencia.

Según Freixas y Rochet (1997) tanto la empresa como el banco buscan satisfacer una función objetivo sometida a un conjunto de restricciones: a) La empresa, tratará de maximizar una función de beneficios para una determinada función de producción, eligiendo un nivel de inversión "I" y su financiación (servicio financiero demandado); b) Por su parte el banco, maximizará una función de beneficios mediante la oferta del servicio financiero, su mezcla de depósitos y la emisión de títulos.

En esta perspectiva los autores coinciden con Pascual y González (1998). Para ellos, el 
marketing bancario es un conjunto de técnicas de comercialización cuyos elementos básicos son: 1) Existencia de un doble mercado (oferente de fondos prestables-bancoutilizadores de fondos prestables) bajo el principio de la doble disponibilidad (Rodríguez, 1980:135); 2) Fuerte competitividad y asimilación de las innovaciones financieras (debido a la volatilidad de la tasa de interés, el surgimiento de los mercados futuros, de opciones, el surgimiento de las tarjetas de crédito, la internacionalización de los mercados financieros, la titularización entre otros) (Mishkin, 1995: 232 -243) y utilización de las denominadas "ventas cruzadas" (combinación de productos/servicios ofrecidos a un mismo cliente); 3) Existencia de una clientela heterogénea; 4) Confidencialidad en los servicios y; 5) Prestación de servicios a cualquier hora en tiempo real.

\section{Algunos determinantes clave en el desempeño del ejecutivo de negocios bancarios}

En el desempeño de un ejecutivo de negocios en general y el bancario en particular concurren, según Alba y Hutchinson (1987), al menos dos elementos: la dimensión horizontal (o articulación) y la dimensión vertical (concebido como organización jerárquica). La dimensión horizontal hace referencia a la habilidad del vendedor de hacer frente a un sin número de situaciones de venta. Por su parte la dimensión vertical tiene que ver con el uso de sus experiencias para afrontar diferentes situaciones en un acto de venta. Según Leong, Busch y John (1986) empíricamente ambas dimensiones pueden ser operacionalizadas, desde el punto de vista de la elaboración de argumentos de venta en: distinción, contingencia y capacidad de simulación. La distinción, según Bower, Black y Turner (1979), mide el grado en que una acción de venta emprendida por un vendedor es única (en términos de estímulos) y con argumentos únicos en respuesta a variaciones en la situación de venta.

Por otra parte, los vendedores tienen la capacidad de organizar sus argumentos de venta jerárquicamente. Existen situaciones de venta que solamente necesitan cierta secuencia sencilla (estrictamente desplegadas y sucesivamente vinculadas en un orden temporal) de argumentos para cerrar ventas. En contraste, existen secuencias no tan estrictamente desplegadas y que constan de secuencias alternativas para responder a cambios en la situación de venta contingentes.

Por último, los vendedores son capaces de abstraer elementos de diferentes experiencias que les permiten desarrollar argumentos más sólidos de cara al cliente. De esta manera la habilidad que un vendedor pueda desarrollar sobre la base de experiencias de ventas previas, es un elemento clave para el aumento del conocimiento del oficio y que se 
traduce en mejores argumentos de ventas.

En términos del ejecutivo de negocios bancarios, las características de la oferta de servicios bancarios y la heterogeneidad de los clientes, generan múltiples acciones de ventas con argumentos variados. Esta situación se presenta inclusive para un mismo cliente. La razón estriba en que el ejecutivo de negocios realiza, en cada situación de venta, un ensamblaje entre las posibilidades que representa el producto/servicio en términos de precio, las características de los contratos que lo sustentan, las restricciones financieras que impone la organización y los deseos del cliente.

Además, una nota distintiva de este tipo de vendedores es la capacidad de portar consigo un arsenal de argumentaciones que permiten posibles combinaciones de productos/servicios a la hora que el cliente cambie de opinión o proponga una variante no contemplada en el plan inicial de venta. Esto hace del ejecutivo de negocios bancarios una persona diestra en la generación de argumentos y contraargumentos $\underline{5}$, estando en capacidad de generar un conjunto de experiencias de ventas que constituyen un elemento esencial en el éxito de su gestión antes y después de la venta.

Ahora bien, el desarrollo de estrategias de venta por parte del ejecutivo de negocios en el contexto financiero implica, entre otras cosas, analizar los elementos operativos que permiten el despliegue de la dimensión horizontal o vertical. Tales elementos se sustentan en una serie de características que, en este medio, posee el ejecutivo de negocios; sus fuentes de información, los criterios para seleccionar clientes, los cronogramas de visitas, la gama de productos/servicios ofrecidos y las actividades después de haber realizado la venta.

\section{Análisis de los resultados}

\subsection{Estrategias funcionales de ventas personales}

Los ejecutivos de negocios bancarios expresaron, que las cualidades de carácter personal más importantes están referidas al aspectos comunicacional, y de personalidad al momento de contactar al cliente. La Tabla 1, muestra aquellas cualidades que según los entrevistados debe poseer todo ejecutivo de negocios bancarios para asegurar el comienzo y consolidación relaciones comerciales entre el banco y el cliente. En primer lugar, la cualidad más apreciada, en un $50 \%$ de los casos es: ser comunicativo. En opinión de los consultados, esto implica que el ejecutivo debe estar en capacidad de escuchar lo que el cliente desea, sus dudas, objeciones o juicios. De igual manera consideran que la 
comunicación implica que escuchar es seguida por la respuesta (entendida como propuesta o contrapropuesta) al cliente.

Otro aspecto valorado por quienes fueron entrevistados es la proactividad. Los ejecutivos de negocios están claros en el hecho que el cliente, por lo general a nivel corporativo, no demanda servicios bancarios clásicos sino que, por lo general demanda servicios combinados (por ejemplo, cartas de créditos con arrendamiento financiero con o sin opción de compra). La proactividad está en el hecho que el ejecutivo trata de anticipar (previo estudio del tipo de empresa, el sector económico y su experiencia) aquella mezcla que cumpla los requerimientos. Esto significa, que mientras el cliente explica, más o menos lo que desea, el ejecutivo piensa (despliegue de la articulación y/o la organización jerárquica) rápidamente aquella combinación de servicios que se ajuste tanto a esos deseos como a las políticas financieras del instituto crediticio al cual presta servicios en el sentido de Alba y Hutchinson (1987).

Es de hacer mención a otras dos cualidades que fueron reseñadas por los entrevistados: Por un lado, el $18,75 \%$ consideró que el ejecutivo debe ser amable (simpático y agradable) y el $12,50 \%$ manifestó que el ejecutivo debe ser algo tolerante. La tolerancia, en muchos casos indicaron los entrevistados, es una cualidad que debe ser puesta de manifiesto para los tipos de personalidades de los clientes tales como: la persona minuciosa y prolija (el displástico), el suspicaz, el indeciso o el grosero.

Las anteriores cualidades que se esperan de un ejecutivo de negocios, especialmente del sector petrolero, fueron consideradas separadamente. Es importante señalar que más del $31 \%$ de los entrevistados expresaron que un ejecutivo de negocios debe dominar todas esas cualidades. La pregunta que se podría formular en estos momentos es porqué existen estas diferencias entre consideraciones globales y parciales, la respuesta está en el hecho que no todo el mundo puede dominar de forma balanceada todas las cualidades. Los ejecutivos de negocios entrevistados reconocieron que existen momentos en los cuales por ejemplo, ser proactivo en la venta es más importante que la simpatía. Lo anterior respalda el argumento de Bower, Black y Turner (1979) en el sentido que una acción de venta emprendida por un vendedor es única y con argumentos únicos en respuesta a variaciones en la situación de venta. Asimismo, se demuestra que el ejecutivo debe desplegar argumentos de ventas con secuencias flexibles (Leong, Busch y John, 1986). 


\begin{tabular}{lcc} 
& Tabla 1 \\
$\begin{array}{c}\text { Cualidades que debe tener el } \\
\text { ejecutivo de negocios bancarios }\end{array}$ \\
\hline Concepto & Frecuencia & $\%$ \\
\hline Comunicativo & 8 & 50.00 \\
Amable & 3 & 18.75 \\
Tolerante & 2 & 12.50 \\
Proactivo & 6 & 37.50 \\
Todas las anteriores & 5 & 31.25 \\
\hline Fuente: datos de la encuesta a ejecutivos de nega \\
dios bancarios en Maracaibo (Verezuela) \\
Cálculos propios.
\end{tabular}

Ahora bien, una de las cosas que el ejecutivo de negocios utiliza para localizar (de la forma más precisa posible) a sus potenciales clientes, es diferentes fuentes de información disponibles en forma individual o conjunta (Tabla 2 ).

Como se puede apreciar, el ejecutivo no necesariamente utiliza una sola fuente de información. El 56, 25\% de los ejecutivos de negocios entrevistados, manifestaron utilizar todas las opciones de la Tabla 2, es decir, al no existir una fuente única, al cruzar esos listados, puede localizar algún cliente potencial. Sin embargo, aunque esta pareciera ser la estrategia, el $43,75 \%$ apeló a una fuente en particular. De esas fuentes, las más utilizadas fueron: 1) las elaboradas por empresas especializadas en investigación comercial (al estilo de Dum \& Bradstreet) con un $18,75 \%$; 2) la Guía Industrial de Venezuela, el Directorio de la Cámara Petrolera, el Directorio de la Cámara de Comercio o el listado de clientes de la agencia (listado básicamente de cuentas corrientes, de pagarés o de líneas de crédito no utilizados) con un $25 \%$ en cada caso; 3 ) en un menor porcentaje, se hizo uso de los estudios de mercado y la guía telefónica.

En el proceso de captar cuentas corporativas (industrial, agroindustrial o petrolero), es indispensable conocer alguna forma para acceder y sobre todo seleccionar a esos clientes. La Tabla 3 muestra los criterios que los ejecutivos de negocios emplean entrevistados para seleccionar a sus clientes. Como se podrá apreciar, la prudencia aconseja que la selección de clientes dependa de una serie de criterios combinados. Es así como el 50\% de los entrevistados, seleccionaron a sus clientes sobre la base: del capital social (existen bancos, según información de los ejecutivos, donde los montos mínimos varían entre medio y un millardo de Bolívares), tamaño de la nómina (tanto en número de empleados y trabajadores y el volumen total en términos monetarios), volumen de ventas (al igual que en el capital social, cada banco establece mínimos de total de ventas "netas" (ventas 
brutas menos devoluciones rebajas, descuentos entre otras deducciones) para ser considerados como asignable a un ejecutivo de negocios petrolero), potencial de pago a corto plazo (medido inicialmente por los indicadores anteriores y índices de solvencia, liquidez, saldos compensatorios o rotación del capital de trabajo neto, es decir, el estudio de los componentes de los activos circulantes en relación con los pasivos circulantes tanto en términos monetarios como en términos de días en manos de cuentas por cobrar, pagar e inventarios de mercancías).

\section{Tabla 2}

Fuentes de información de clientes potenciales usadas por los Ejecutivos de Negocios Bancarios

\begin{tabular}{lcc}
\hline Concepto & Frecuencia & $\%$ \\
\hline Estudios de mercado & 1 & 6.25 \\
Guia industrial & 4 & 25 \\
$\begin{array}{l}\text { Directorio de la } \\
\text { Camara de Comercio }\end{array}$ & 4 & 25 \\
$\begin{array}{l}\text { Directorio de la } \\
\text { Camara petrolera }\end{array}$ & 4 & 25 \\
$\begin{array}{l}\text { Registro electrónico } \\
\text { de clientes }\end{array}$ & 2 & 12.5 \\
$\begin{array}{l}\text { Listado de clientes } \\
\text { de crédito }\end{array}$ & 4 & 25 \\
$\begin{array}{l}\text { Guia telefónica } \\
\text { Informadoras } \\
\text { comerciales }\end{array}$ & 1 & 6.25 \\
$\begin{array}{l}\text { Todas las anteriores } \\
\text { Otras }\end{array}$ & 9 & 18.75 \\
$\begin{array}{l}\text { Fuente: datos de la encuesta a ejecutivos de nega } \\
\text { cios bancarios en Maracaibo (Venezuela) } \\
\text { Calculos propios. }\end{array}$ &
\end{tabular}

Lo anterior es lo que permitirá el establecimiento de transacciones, relaciones a largo plazo Kotler (1996) y de satisfacer una función objetivo (maximización del beneficio) sometida a un conjunto de restricciones financieras (Freixas y Rochet, 1997).

Es interesante destacar, que el $50 \%$ de los ejecutivos de negocios entrevistados utiliza todas las opciones anteriores para seleccionar su clientela. Este resultado refleja que estos ejecutivos de negocios evalúan todos los aspectos financieros posibles de las empresas con la finalidad de introducir una mayor cantidad de productos/servicios (tanto de captaciones como de colocaciones) y crear más vínculos comerciales. Es de hacer notar, que una mayor consideración de factores de carácter financiero económico a la hora de 
evaluar al posible cliente es iniciativa del ejecutivo de negocios.

Un 18.8\% seleccionó únicamente como criterio el capital social y volumen de ventas. Mientras que dos ejecutivos de negocios seleccionaron solamente el tamaño de la nómina o el potencial de pago a corto plazo. Estos ejecutivos de negocios declararon que los criterios de capital social, volumen de ventas o tama ño de la nómina eran establecidos por la dirección general d sus bancos. En consecuencia, se aprecia que la estrategia-funcional formal prevalece sobre la emergente.

Resumiendo, gran parte de las estrategias funcionales de captación de clientes son de carácter emergente corroborando lo que establecía Mintzberg (1994) acerca de la existencia, en este caso, en el área de ventas bancario de estrategias que se escapan de la lógica de la planificación formal del banco.

La selección y captación de los clientes, debe ser complementado por una frecuencia de visitas. El 50\% de los ejecutivos entrevistados, expresaron que realizan al menos una visita al mes a sus clientes. Por su parte un $25 \%$ contestó, que realizan visitas una vez a la semana. Dos ejecutivos realizan una visita trimestralmente y otros dos de manera frecuente (aunque sin especificar una frecuencia definida).

Tabla 3

Criterios adoptados por los ejecutivos de negocios bancarios para seleccionar clientes

\begin{tabular}{|c|c|c|}
\hline Concepto & Frecuencia & $\%$ \\
\hline Capital Social & 3 & 18.80 \\
\hline $\begin{array}{l}\text { Tamaño de la } \\
\text { nómina }\end{array}$ & 1 & 6.25 \\
\hline Volumen de ventas & 3 & 18.80 \\
\hline $\begin{array}{l}\text { Potencial de pago } \\
\text { a corto plazo }\end{array}$ & 1 & 6.25 \\
\hline $\begin{array}{l}\text { Todas las } \\
\text { anteriores }\end{array}$ & 8 & 50.00 \\
\hline Totales & 16 & 100,00 \\
\hline \multicolumn{3}{|c|}{$\begin{array}{l}\text { Fuente: datos de la encuesta a ejecutivos de nego } \\
\text { cios bancarios en Maracaibo (Venezuela) } \\
\text { Cálculos propios. }\end{array}$} \\
\hline
\end{tabular}

Según los ejecutivos, son diversas las razones para establecer o alterar la frecuencia de visitas: cuando la frecuencia es elevada, es porque el cliente no ha sido captado para una cuenta que reporte un alto retorno; ese cliente representa un volumen de negocios 
importante para el banco (sobre todo para el futuro al poder ser cruzado con otros productos/servicios); una vez al mes, implica visitas de mantenimiento, es decir, la cuenta ha sido captada y las visitas son para vigilar las nuevas necesidades del cliente o nuevos productos/servicios que pueden ser ofrecidos; frecuencias muy bajas significan que los clientes no han sido captados para ningún producto/servicio (son los clientes con más objeciones, dudas o suspicacias acerca de la capacidad del banco para brindar el servicio que promete o clientes con una relación de "fidelidad" con otro banco), pero tienen relaciones indirectas (por ejemplo sus proveedores tienen cuentas con el banco del ejecutivo de negocios) con el instituto bancario.

Con referencia al tipo de productos/servicio ofrecido, en la Tabla 4 se puede observar que los ejecutivos de negocios en un $75 \%$, ofrecen todos los productos/servicios que posee su instituto bancario. Sin embargo, existen ejecutivos que declararon ofrecer productos/servicios por separado. El $25 \%$ ofrece cuentas corrientes, participaciones y fideicomisos; el $18.75 \%$ cuentas de ahorro, créditos comerciales, créditos hipotecarios y fianzas, el $12.50 \%$ créditos al consumidor, depósitos a plazo fijo y cartas de crédito y un $6.25 \%$ créditos agropecuarios.

Se puede observar, que los ejecutivos de negocios ofrecen una gran variedad productos/servicios de la banca comercial y universal a sus clientes. Pero al analizar la oferta individualizada, según el número de respuestas, se observa que 17 respuestas corresponden a ofertas referidas a productos/servicios para generar ingresos financieros a las empresas; mientras que 14 respuestas corresponden a aquéllos relacionados con ingresos financieros para los institutos bancarios. 


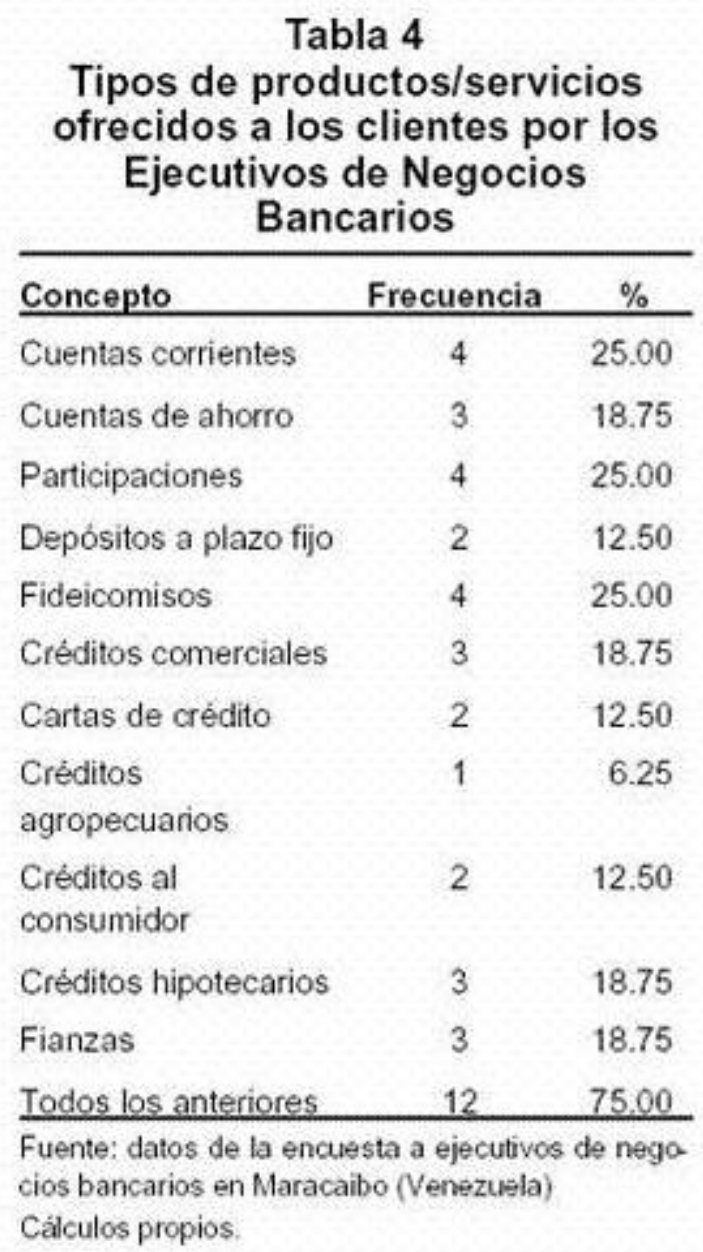

Estos resultados muestran algunos indicios de: 1) La fuerte competencia interbancaria por la captación de clientes por lo cual los bancos se quitan clientes unos a otros, utilizando la oferta de productos/servicios de ahorro como estrategia de captación, un intenso contacto telefónico diario para captarlos (Tabla 5) y 2) La existencia de un mercado donde predomina el enfoque de la demanda (Fernández, 1977).

Lo que es importante destacar en este punto son las combinaciones que hacen los ejecutivos de negocios de los diferentes productos/servicios mencionados en la Tabla 4. Por ejemplo, algunos ejecutivos ofrecían financiamiento en Dólares del banco ubicado en la ciudad de Miami con garantía de un inmueble ubicado en Venezuela y valuado también en dólares. Para respaldar la operación, se procedía a contratar una póliza de seguro con la empresa filial del grupo financiero en dólares, tal operación podría servir para financiar capital de trabajo en Venezuela. Otro ejecutivo explicó la realización de operaciones con las denominadas cartas de crédito back to back (en este caso una carta de crédito de exportación con los mismos términos de la carta de crédito de importación para respaldar la operación de exportación desde Venezuela) y financiado con descuentos de giros. Lo 
novedoso estribó en el hecho que se incluyeron términos adicionales de negociación referidos a la necesidad de presentar certificación de embarque y de una empresa especializada como Bureau Veritas. Por último, otros ejecutivos en el sector petrolero destacaron la realización de operaciones combinadas por medio de cesiones de crédito (préstamos a las empresas con garantía de facturas pendientes de cobro), cesiones de contratos (préstamos con garantía de contratos de servicios otorgados por Petróleos de Venezuela), valuaciones (préstamos sobre la base del valor de inmuebles, maquinaria o bienes previamente valuados) y órdenes de compra (préstamos garantizados con órdenes de compra de mercancías firmados y aprobados).

Estos y otros ejemplos indican que la institución financiera no está en capacidad de ordenarle a los ejecutivos de negocios el tipo de operaciones híbridas, las condiciones específicas y la forma de ofrecerla a los clientes potenciales, es por esto que el ejecutivo de negocios toma una serie de decisiones de carácter funcional. Por supuesto que el ejecutivo de negocios debe tomar en consideración una serie de elementos de política, como por ejemplo en materia de tasas de interés, comisiones, condiciones de negociación, etc. Sin embargo, el ejecutivo de negocios cuenta con herramientas de persuasión para que el banco acepte aspectos de la negociación que, saliéndose de las políticas y estrategias operativas establecidas, representen una oportunidad de negocios para la institución $\underline{6}$.

\section{Tabla 5}

Número de contactos telefónicos realizados por los Ejecutivos de Negocios Bancarios

\begin{tabular}{lcc}
\hline Concepto & Frecuencia & $\%$ \\
\hline Diariamente & 14 & 87.50 \\
Una vez a la & 2 & 12.50 \\
semana & & \\
Totales & 16 & 100.00 \\
\hline $\begin{array}{l}\text { Fuente: datos de la encuesta a ejecutivos de nego- } \\
\text { cios bancarios en Maracaibo (Venezuela) } \\
\text { Calculos propios. }\end{array}$
\end{tabular}

No obstante, los ejecutivos de negocios del sector entrevistados ofrecen productos/servicios adicionales (ver Tabla 6). En primer lugar, el 75\% de los entrevistados señalaron que se ofrecen combinadamente: pago de nóminas a empleados y en forma automáticas, pago a proveedores automáticamente y operaciones internacionales. En segundo lugar, considerados individualmente, el pago automático de nóminas y proveedores es ofrecido por el por el $18.75 \%$ de los entrevistados y el $12.50 \%$ con el 
pago a empleados (por vía de la caja principal) y operaciones internacionales (convenio de la Asociación Latinoamericana de Integración, transferencias, cobranzas internacionales entre otras).

\subsection{Estrategias funcionales de postventa y asesorías}

Ahora bien, el proceso de venta no se queda solamente en hacer que un cliente abra una determinada cuenta de depósito o contrate un financiamiento por medio de valuaciones. Los ejecutivos de negocios están conscientes, de acuerdo a sus respuestas, que debe darse importancia a los aspectos del servicios tales como: El tiempo de respuesta a las inquietudes y la forma de llevar el control de la postventa de productos/servicios.

El $87.5 \%$ de los ejecutivos entrevistados manifestaron atender de inmediato las solicitudes de los clientes y el resto manifestó atenderlo en forma mediata. La atención inmediata se refiere en primer lugar que las inquietudes o quejas de los clientes es recogida procesada al mismo tiempo y se explica al cliente el tiempo aproximado de su solución y hasta, cómo lo harán, en segundo lugar, reconocen que no todas las preocupaciones y dudas de los clientes se pueden resolver efectivamente en cuestión de minutos e inclusive horas. La atención mediata se refiere a que existe un intervalo de tiempo significativo (más de un día) entre la recogida de la información del cliente y su procesamiento. No se debe olvidar que las operaciones de la banca siguen una secuencia de pasos y que los mismos no son controlados totalmente por el ejecutivo. Mucho de esos pasos dependen de áreas diferentes en función del producto/servicio contratado y en algunos casos el centro de control y decisión se encuentra fuera de la región (porque quizá el banco tiene su sede principal fuera de la ciudad). 


\begin{tabular}{l}
$\quad \begin{array}{l}\text { Tabla } 6 \\
\text { Tipos de servicios adicionales } \\
\text { ofrecidos por los Ejecutivos } \\
\text { de Negocios Bancarios }\end{array}$ \\
$\begin{array}{l}\text { Concepto } \\
\text { Frecuencia }\end{array}$ \\
$\begin{array}{l}\text { Pago a nóminas } \\
\text { automáticas }\end{array}$ \\
$\begin{array}{l}\text { Pago a empleados } \\
\text { Pago a proveedores }\end{array}$ \\
$\begin{array}{l}\text { automáticamente } \\
\text { Operaciones } \\
\text { internacionales }\end{array}$ \\
$\begin{array}{l}\text { Todas las anteriores } \\
\text { Fuente: datos de la encuesta a ejecutivos de nego. } \\
\text { cios bancarios en Maracaibo (Venezuela) } \\
\text { Calculos propios. }\end{array}$ \\
\hline
\end{tabular}

En consecuencia, el ejecutivo de negocios realiza una serie de acciones y decisiones que pueden escapar del sistema formal de trabajo y planificación del banco. El ejecutivo puede seguir una estrategia funcional de prepararse como operador del sistema de procesamiento de transacciones (para postear notas de cébito o crédito por ejemplo), hacer un trato con el gerente de crédito de una agencia (el ejecutivo le abre una cuenta al cliente o le adscribe un crédito a esa agencia) para usar la infraestructura comunicacional (terminales y teléfonos) y de atención al cliente para captar una cuenta cercana a esa agencia, en este caso el gerente de la agencia y el ejecutivo de negocios ganan, por un lado, el gerente capta una cuenta y/o un crédito y, por el otro lado, el ejecutivo evita dar al cliente una respuesta prolongada en el tiempo.

Desde el punto de vista del control de la postventa, el ejecutivo de negocio hace un uso intensivo de la visita personal y del teléfono, sin embargo, como se puede apreciar en la Tabla 7, el Internet (correo electrónico principalmente) aparece también como forma de llevar un control en tiempo real de las acciones de postventa con el cliente, es decir, la recepción de reclamos, su control y la forma de canalizar la solución por medio de otras instancias operativas y administrativas del banco.

En la visita personal, el ejecutivo de negocios puede palpar en el lugar las reacciones del cliente ante los productos/servicios ofrecidos. En caso de problemas, él se ve obligado a instrumentar otras estrategias para ofrecer una respuesta más oportuna y rápida, es decir, constantemente está evaluando, como lo expresó uno de los ejecutivos entrevistados, "Ios canales disponibles para procesar quejas, objeciones y ofrecer soluciones". En este punto es interesante destacar que los ejecutivos de negocios más 
hábiles dicen que se debe poseer los códigos de cajeros (eso se lleva en un libro especial), los códigos de transacciones o el Códigos de Cuentas de la Superintendencia Bancaria. La razón de tal afirmación estriba en que: “. . . con frecuencia se presentan reclamos, por ejemplo, en materia de cobro de comisiones o de tarifas a cuentas que tienen condiciones especiales, es decir, cuentas exoneradas de cargos por servicios. Lo cierto es que en el estado de cuenta aparece una serie de números y el cliente pregunta por su significado. Al tener disponibles los códigos respectivos, se puede saber quién hizo el cargo y por cuál cajero. El paso siguiente sería llamar al respectivo departamento y verificar la realización de la transacción. Resulta ser, que puede darse el caso que en ese departamento no existía la información sobre las condiciones especiales o se hizo por error...". El problema no es la obtención de los códigos, expresa un ejecutivo entrevistado: "Sino su actualización, debido a que los bancos rotan constantemente el personal y constantemente se emiten circulares referidas a las operaciones y el procesamiento contable de determinadas transacciones. Para solventar tal situación, se entablan relaciones con los ejecutivos a cargo para un envío del material actualizado".

\section{Tabla 7}

Medios de control postventa de los productos/servicios vendidos. Utilizados por los Ejecutivos de Negocios Bancarios

\begin{tabular}{lcc}
\hline Concepto & Frecuencia & $\%$ \\
\hline Internet & 4 & 25.00 \\
Teléfono & 13 & 81.25 \\
Visita personal & 16 & 100.00 \\
\hline
\end{tabular}

Fuente: datos de la encuesta a ejecutivos de negocios bancarios en Maracaibo (Venezuela)

Cálculos propios.

Con respecto al teléfono como en cualquier institución que maneja dinero es indispensable su disponibilidad. Sin embargo, en las agencias u oficinas principales, su disponibilidad es limitada. Se ha optado por el uso del celular, ya que permite al cliente una localización del ejecutivo en todo momento. Debido al costo que representa para el ejecutivo el uso del celular, los bancos han tenido que aceptar el pago de la cuenta telefónica, pero solamente relacionada con los clientes del banco.

Por último, se abordó la relación entre las asesorías ofrecidas por el banco y las asesorías demandadas por los clientes. Por asesoría del ejecutivo de negocio se entiende, en este trabajo, como la serie de consejos que ilustran al cliente acerca de cuáles decisiones y acciones adoptar y que serían más convenientes para interés de él y del 
banco.

En lo referente al tipo de asesoría ofrecida por los ejecutivos de negocios petroleros, la Tabla 8 muestra, que el $43.75 \%$ de los ejecutivos entrevistados respondió que asesoran en todos los productos/servicios. Sin embargo, considerados individualmente, la distribución se presentó de la siguiente forma: 1) Un 37.5\% brindó asesoría en capital de trabajo y fianzas, el cual se podría entender como aquella relacionada con la capacidad de la empresa para atender a sus compromisos a corto plazo y mecanismos para garantizar financiamientos a la propia empresa o empresas relacionadas; 2) Un $31.25 \%$ de los entrevistados manifestó ofrecer asesorías relacionadas con créditos comerciales y cartas de crédito (principalmente de importación), es decir, relacionadas con financiamientos a corto plazo; 3) Un 25\%, respondió que ofrecían asesorías para importaciones y exportaciones, esto es, trámites para exportar (pre y post embarque) así como explicar el proceso de importación (desde las negociaciones entre comerciantes, el mecanismo de las corresponsalías, las discrepancias, los trámites aduanales y sobre todo el sistema de tarifas y financiamiento que utiliza el banco; 4) Un 18.75\% asesoró en créditos al consumidor y en igual porcentaje en descuento de giros (e inclusive el uso y ventajas de la matrícula de descuento) y 5) El $6.25 \%$ de los ejecutivos especializaron sus ofertas mediante créditos con recursos del Estado como Foncrei y Bancoex (Es decir, por un lado un financiamiento cuya tasa de interés en cierto sentido es más baja que la banca no estatal y para aquellos proyectos que por el monto y riesgo cambiario principalmente exige un alto nivel de cobertura), créditos hipotecarios (con garantía hipotecaria ya sea de activos inmuebles localizados en el país o de activos inmuebles localizados en el exterior).

Es importante destacar, que los bancos comerciales no asesoran al cliente en materia financiera, la costumbre ha sido el evaluar el potencial de pago del cliente y si es una buena cuenta se le brinda financiamiento, pero en el ámbito corporativo, los ejecutivos han descubierto que la asesoría es una herramienta poderosa para establecer relaciones perdurables en el tiempo con los clientes. Esto no significa que el ejecutivo de negocios hace las veces de un gerente de finanzas de una empresa o consejero de tesorería (nacional o internacional). Más bien, como analista de estados financieros, el ejecutivo de negocios se percata de las debilidades y fortalezas de la empresa para acceder a determinado financiamiento. En este sentido, el ejecutivo de negocios usa como estrategia funcional su conocimiento acerca de la empresa para proponerle una paquete financiero y de servicios que sea ventajoso para la empresa y que represente también ventajas para el banco. Lo complicado estriba en el hecho que el ejecutivo de negocios debe balancear ambos polos. 


\section{Tabla 8}

Tipo de asesoria ofrecida por los Ejecutivos de Negocios Bancarios y demandada por los clientes petroleros

\begin{tabular}{lcccc}
\hline Tipos de Asesoria & Asesoria ofrecida & $\%$ & Asesoria demandada & $\%$ \\
\hline Capital de trabajo & 6 & 37.5 & 10 & 62.50 \\
Importaciones & 4 & 25 & 7 & 43.75 \\
Exportaciones & 4 & 25 & 3 & 18.75 \\
Créditos comerciales & 5 & 31.25 & 6 & 37.50 \\
Cartas de crédito & 5 & 31.25 & 9 & 56.25 \\
Créditos con recursos & 1 & 6.25 & 2 & 12.50 \\
del Estado & 3 & 18.75 & 3 & 18.75 \\
Créditos al consumidor & 1 & 6.25 & 2 & 12.50 \\
Créditos hipotecarios & 6 & 37.5 & 7 & 43.75 \\
Fianzas & 3 & 18.75 & 5 & 31.25 \\
Descuentos de giros & 7 & 43.75 & 2 & 12.50 \\
\hline Todas las anteriores & & & & \\
\hline
\end{tabular}

Fuente: datos de la encuesta a ejecutivos de negocios bancarios en Maracaibo (Venezuela) Cálculos propios.

El ejecutivo está consciente de las limitaciones y restricciones de orden legal, de orden económico y de políticas institucionales; sin embargo, cuenta con una serie de estrategias interna y externas para lograr su cometido. En primer lugar, puede realizar estudios de los estados financieros sin necesidad que lo haga la respectiva unidad de la dirección general, lo que ahorra tiempo en la tramitación del crédito. En segundo lugar, al conocer la mecánica de tramitación y de toma de decisiones del comité de crédito corporativo, está en mayor capacidad de promover su caso en forma más rápida y con un menor número de objeciones posibles por parte del comité (siempre en el supuesto de que el cliente sea una buena oportunidad). En tercer lugar, puede anticipar, previa negociación con el cliente las garantías de refuerzos que el banco acostumbra solicitar para determinados clientes (sobre todo con poca experiencia con el banco). En cuarto lugar, al conocer las técnicas, normas de liquidación, el saber imprimir un sentido de urgencia y contar con firma autorizada, está en mejor posición de procesar la liquidación.

En lo referente a la asesoría demandada, la preferencia de los clientes se concentró: En primer lugar, en la administración del capital de trabajo neto (o sea, en el ciclo de comercialización de la empresa); en segundo lugar, en la apertura de créditos; y más concretamente en las cartas de crédito (de importación especialmente); en tercer lugar y relacionado a lo anterior, con las asesorías en trámites de importación (aspectos fiscales, 
financiamiento y transporte entre otros) y de la utilización de las fianzas como modalidad de garantizar financiamiento; en cuarto lugar, se encuentra la asesoría en créditos comerciales en el $37.5 \%$ de los casos y los descuentos de giros con el $31.25 \%$ de las respuestas ofrecidas por los ejecutivos; en quinto lugar se encuentra, con un $18.75 \%$ los créditos personales (especialmente con tarjetas de crédito) y créditos relacionados con las exportaciones (financiamiento de pre y post embarque); por último en un $12.5 \%$ de los casos, se encuentra la solicitud de créditos para financiamiento por medio de fondos de organismos de financiamiento del sector público, créditos con garantía hipotecaria (lo que refleja el poco interés de los clientes en otorgar este tipo de garantía para respaldar los financiamientos) y el ofrecimiento del ejecutivo de negocios de toda la gama de productos/servicios mencionados con anterioridad.

Al comparar lo ofrecido con lo demandado, se pudo observar lo siguiente:

1. Mientras que los ejecutivos de negocios tienden a preferir una oferta conjunta de los productos/servicios del banco, los clientes petroleros son selectivos. Esto en una primera etapa. Cuando el ejecutivo de negocios plantea que varios servicios pueden combinarse, entonces el cliente deja la demanda selectiva y se concentra en las ventajas del paquete ofrecido.

2. Los Ejecutivos de negocios ponen más énfasis en aquellos productos/servicios más relacionados a operaciones activas (exportaciones, financiamiento, cartas de crédito).

3. Los ejecutivos de finanzas de las empresas o analistas de tesorería ponen mucho énfasis en que las tarifas sean aceptables a sus intereses, por tal razón, según explicaron algunos de los ejecutivos consultados, se contactan simultáneamente varios bancos por vía telefónica, por ejemplo, para seleccionar aquel banco que para unos servicios determinados ofrece mejores condiciones de tarifas o comisiones.

4. A medida que el ejecutivo de negocios establece un patrón del cliente, está en capacidad de ofrecer productos/servicios más relacionados directamente con el negocio petrolero y de cruzarlos con otros previamente adquiridos por el cliente.

\section{Conclusión}

Lo anteriormente expuesto, pone de relieve la existencia de estrategias funcionales emergentes de ventas por parte de los ejecutivos de negocios bancarios en el sentido de Mintzberg (1978) y (1994), por medio de la puesta en marcha de una serie de elementos de carácter personal y operativo.

Los ejecutivos de negocios entrevistados consideran que se debe combinar una serie de 
cualidades personales (trato, acogida, habilidad para entender lo que el cliente requiere en un momento dado) y experiencia tanto de orden legal (principios de los contratos bancarios que rigen los productos/servicios), habilidades operativas (manejo de los códigos de cuentas de las transacciones empleadas por el sistema de computación del banco, los códigos de las cuentas que se movilizan para expresar los cargos y abonos de las transacciones realizadas o quiénes son las personas que realizan esas labores). Esto no significa que el ejecutivo de negocio debe ser un experto en los sistemas operativos y contables del banco, sino que su conocimiento puede darle claves sobre las ventajas, inconvenientes, limitaciones y restricciones de esos sistemas y estar en capacidad de ofrecer unos servicios adecuados en el sentido de advertir adecuadamente al cliente que en cierto momento puede afrontar ciertos problemas y los posibles cursos de acción (Schauer, 1997).

Con lo anterior, el ejecutivo de negocios busca en las fuentes de información (internas o externas) los clientes potenciales o los actuales, para ofrecer los productos/servicios del banco. Ahora bien, la captación de clientes implica la realización de una serie de actividades no incluida en la planificación y organización formal del banco, sino que se vale de ella: realizar análisis financieros, uso de sus conocimientos acerca de la mecánica de tramitación, aprobación y liquidación de créditos para captar y/o mantener clientes, anticipar los recaudos y las garantías de refuerzos que el banco acostumbra solicitar. En otras palabras, el ejecutivo de negocios se convierte en un departamento de gestión de recursos internos para alcanzar metas de captación y colocación e recursos del banco aunque este, en determinados momentos, como lo expresaron algunos ejecutivos de negocios, la institución se oponga consciente o inconscientemente.

\section{Notas}

1. En este trabajo se equipara el ejecutivo de negocios bancarios con el denominado vendedor directo de productos intangibles según la clasificación de Lambin inspirada en McMurray. Según este autor, es un vendedor de productos tangibles o de productos intangibles, para los cuales la creatividad de la venta y de la estrategia de comunicación juegan un papel importante. Se debe señalar que los 16 ejecutivos de negocios constituyeron el marco muestral considerado.

2. Es interesante destacar, que Ansoff establecía una vinculación intertemporal entre las actividades- productosmercados y su proyección futura. Los componentes que garantizarían esa vinculación son: el ámbito, las competencias distintivas, las ventajas comparativas y la sinergia.

3. El uso que en este trabajo se hace de las estrategias emergentes se refiere a las de carácter funcional. Según 
Hofer y Schendel (1985), la distribución de los cuatro componentes de la estrategia adquiere las siguientes características: 1) Al ámbito le corresponde el desarrollo del producto; 2) Las competencias distintivas están relacionadas con el área funcional; 3) La ventaja competitiva está relacionada con la capacidad de productos concretos para competir; 4) La sinergia está dentro de las funciones. Por otra parte, las decisiones de política funcional más importantes son sobre: promoción, precios, personal y programación de la producción en función de su ciclo de vida.

4. Un bien público es aquél que cuando se provee a un individuo, se hace disponible para otras personas sin costo adicional. El suministro de bienes públicos es un problema de toma de decisiones políticas, que se justifica por la imposibilidad de exclusión y el consumo no-rival. En consecuencia, el Estado los suministra ya sea por el cobro de impuestos, endeudándose o con la creación de dinero. Sin embargo, el Estado debe afrontar la existencia de coaliciones de personas (consumidores), que son racionales y egoístas en el sentido de que ellos realizan acciones que son evaluadas en función de las consecuencias que tales acciones tienen para lograr la maximización de sus intereses en términos de coste- beneficio (Olson, 1999).

5. El ejecutivo de negocios se encuentra fuertemente respaldado por su capacidad para comunicarse (capacidad para hablar y ver, pero sobre todo, de escuchar y observar). Ritter (1992), afirma que esta capacidad consta de tres elementos: el lenguaje corporal, la acción vocal y las palabras. El lenguaje corporal, algunas veces denominado lenguaje no verbal, comprende los ademanes, la postura, la presentación personal. La acción vocal hacen referencia: al timbre (personalidad del sonido), la modulación (por medio de las inflexiones que permite generar matices) y el volumen de voz (capacidad para hacer audible su voz). Por último, el uso de las palabras adecuadas en el discurso de venta desde el punto de vista semántico (desde la perspectiva de su significado) y del contexto en la situación de venta. Por otra parte, en lo que concierne a habilidades, su entrenamiento le ayuda a: Argumentar mejor en la venta; Vencer objeciones de los clientes; Reconocer señales de compra del cliente; Saber hacer la iniciación de la venta; Saber realizar el cierre de la venta; y Hacer la venta extra.

6. La experiencia ha indicado, según los ejecutivos entrevistados, que estas estrategias funcionales emergentes son implementadas contando con la aprobación del respectivo comit é de crédito, de la respectiva vicepresidencia ejecutiva o de la junta directiva de la institución financiera. Aunque estas estrategias de carácter funcional rara vez pasan a formar parte de una estrategias formal a nivel operativo o directivo, se convierten en acciones y decisiones que se aplicarán a casos similares siempre y cuando reúnan una serie de condiciones tales como: tipo de cliente, experiencia de crédito, compensación, monto y tipo de las cargas directas o indirectas, entre otras.

\section{Referencias Bibliográficas}

1. Anderson, R.E.; Hair, J.F y Bush, A.J. (1995), Administración de Ventas. McGraw Hill. Eguna edición. M éxico, D. F.

2. Andrew, K. (1995), Marketing de los servicios bancarios y financieros. Ediciones 
Deusto. Pp 190.

3. Andrew, Kenneth (1971), The Concept of Corporate Strategy. Dow-Jones-Irwin. Homewood. Illinois.

4. Ansoff, Igor (1965), Corporate Strategy: An Analytic Approach to Business Policy for Growth and Expansion. McGraw Hill. New York.

5. Badoc, Michel (1976), El Mercadeo Bancario. Editorial Tecniban, S.A. Madrid. Pp. 338.

6. Chandler, Alfred (1962), Strategy and Structure: Chapters in the History of the American Enterprise. Cambridge, Mass.: MIT Press.

7. Cobra, Marcos (2000), Marketing de Servicios. Segunda edición. McGraw-Hill Interamericana, S.A. Bogotá. Pp. 255.

8. Cobra, Marcos y Zwarg, Flavio (1991), Marketing de Servicios. McGraw-Hill Interamericana, S.A. Bogotá. Pp. 280.

9. Churchill, Gilbert A., Jr; Ford, M Neil; Hartley, Steven W. y Walker, C. Orville, Jr (1985), “The Determinants of Salesperson Perfomance: A Meta Analysis”. Journal of Marketing Research (may). Chicago. Pp. 103-118.

10. Churchill, Gilbert A., Jr, Ford, M Neil, Hartley, Steven W. y Walker, C. Orville., Jr (1985), “Dirección de ventas”. Promociones Jumerca. Valencia. Pp. 230.

11. Drew, Stephen (1995), “Accelerating Innovation in Financial Services". Long Range Planning. Volume 28. No. 34. London. Pp.11-21.

12. Ennew, Cristine; Watkins, Trevor y Wrigth, Mike (1990), Mercadeo estratégico de servicios financieros. Fondo Editorial Legis. Bogotá. Pp. 242.

13. Freixas, Xavier y Rochet, Jean-Charles (1997), Economía Bancaria. Antoni Bosch editor y el Banco Bilbao Vizcaya Argentaria. Madrid. Pp. 378.

14. Fruhan Jr, William E. (1979), "Financial Strategy: Studies in the Creation Transfer and Destruction of Shareholder Value". Homewood. Illinois: Irwing. 
15. Grande, Idelfonso (1992), "Dirección de Marketing". Editorial McGraw Hill/Interamericana de España, S.A.U. Madrid. Pp. 401.

16. Grubbs, Ray y Reidenbach, Eric (1993), Banca de Excelencia.

17. Hernández, R. Garrido (1977), “EI Marketing”. Editorial Index. Madrid. Pp. 151.

18. Hernández, Sampieri Roberto; Collado, Carlos y Lucio, Pilar (1991), “Metodología de la Investigación". Segunda edición. McGraw Hill. México D.F. Pp. 505.

19. Hofer, Charles W. y Schendel, Dan (1985), Planeación estratégica: Conceptos analíticos. Editorial Norma, S. A. Santafé de Bogotá. Pp. 228.

20. Instituto Financiero Ejecutivo (IFE) (1992), Gestión Eficaz de Servicios Financieros. Caracas. Pp. 2.

21. Kotler Philip y Armstrong, Gary (1998), Fundamentos de Mercadotecnia. Editorial Prentice-Hall Hispanoamericana, S.A. Naucalpan de Juárez. Pp. 585.

22. Kotler, Philip (1996), Dirección de Mercadotecnia: análisis, planeación, implementación y control. Editorial Prentice-Hall Hispanoamericana, S.A. Naucalpan de Juárez. Pp. 800.

23. Kotler, Philip y Armstrong, Gary (1989), Mercadotecnia. Editorial Prentice-Hall Hispanoamericana, S.A. Pp. 825.

24. Lambin, Jean Jacques (1995), Marketing Estratégico. Tercera edición. McGraw Hill/Interamericana de España, S.A. Madrid. Pp. 610.

25. Lambin, Jean-Jacques (1995), Marketing Estratégico. Tercera edición. McGraw-Hill Interamericana de España, S.A. Madrid. Pp. 610.

26. Leigh, Thomas, W. (1987), Cognitive Selling Scripts and Sales Training. Journal of Personal Selling \& Sales Management. No. 7. August. Pp. 39-48.

27. Leong, Meng Siew; Busch, S. Paul y John, Roedder Deborah (1989), Knowledge Bases and Salesperson Effectiviness: A script-Theoretic Analysis. Journal of 
Marketing Research. May vol. XXVI. No. 2 Chicago. Pp. 164-178.

28. Lewis, B. R. (1991), Customer Service in a Mayor United Kingdon Bank. Financial Services Research Centre. Manchester School of Management.

29. Lundy, Donald (1990), “The Benefits of Automation". En: Bank Management. April. Pp. 32-37. Manuales del B.O.D.

30. Mapfre (1996), Marketing y gestión comercial de seguros. Editorial Mapfre. Madrid. Pp. 394.

31. Mcgroldrick, Peter y Steven J., Greenland (1996), Comercialización de servicios financieros. Series Mc Graw Hill. Pp. 388.

32. Mintzberg, Henry (1978), Patterns in Strategic Formulation, Management Science. No. 24. Pp. 934-948.

33. Mintzberg, Henry (1994), Rethinking Strategic Planning Part I: Pitfalls and Fallacies. Volume 27, No. 3 J une. London. Pp 12-21.

34. Mishkin, Frederic (1995), The Economy of Money, Banking, and Financial Markets. Fourth edition. The HarperCollins College Publishers. New York. Pp. 757.

35. Morgan, Robert E.; Severn, Eileen Cronin y Severn, Mark (1995), I nnovation in Banking: New Structures and Systems. Long Range Planning. Volume 28. No. 3. London. Pp. 91-100.

36. Olson, Mancur (1999), Sumary and description of the logic of collective action [En línea]. Http://www.unix/cc/ wmich.edu/corder/pls200ca.[Consulta: 26 de julio de 1999].

37. Pascual, López Joaquín y González, Sebastián Altina (1998), Gestión Bancaria: Los nuevos retos en un entorno global. McGraw Hill/Interamericana de España, S.A.U. Madrid. Pp. 335.

38. Ritter, Dwight (1992), La venta de servicios financieros. Ediciones Deusto, S.A. Bilbao. Pp. 179. 
39. Rodríguez, Sergio Azuero (1990), Contratos Bancarios: su significación para América Latina. Cuarta edición. Biblioteca Felaban. Bogotá. Pp. 708.

40. Schauer, Armin (1997), Verkäufer-Training: Was bringt das wirklich? Marketing Journal. Juni-Juli. No. 3. Hamburg. Pp. 190-192.

41. Stoner, James. A.F. y Wankel, Charles (1989), Administración. Tercera edición. Editorial Pretince Hall Hispanoamericana, S.A. Naucalpan de Juárez. Pp. 826.

42. Swan, W.S (1991), Cómo escoger al Personal Adecuado: Un programa adecuado para hacer la entrevista. Editorial Norma. Santafé de Bogotá.

43. Sujan, R. Harrish; Sujan, Mita y Bettman, James (1988), Knowledge Structure Differences Between More Effective and Less Effective Salespeople. Journal o Marketing Research. No. 25. February. Chicago. Pp. 81-86.

44. Szymanski, David M. (1988), Determinants of Selling Effectiviness: The importance of Declarative Knowledge to the Personal Selling Concept. Journal of Marketing. No. 52. January. Pp. 64-77.

45. Weitz, Barton A. (1981), “Effectiviness of Sales Interactions: A Contingency Framework". Journal of Marketing. No. 45 (Winter). Pp. 85-103. 